\title{
Understanding Requirements of Ubiquitous Application in Context of Daily Life
}

\author{
Naotake Hirasawa ${ }^{1}$, Tomonori Shibagaki ${ }^{1}$, Hideaki Kasai ${ }^{2}$ \\ ${ }^{1}$ Otaru University of Commerce Midori 3-5-21 Otaru, \\ Hokkaido, Japan 047-8501 \\ ${ }^{2}$ NEC Software Hokkaido, Ltd, 28 Kita 8 Nishi 3 Kita-ku Sapporo, \\ Hokkaido, Japan 060-0808 \\ hirasawa@res.otaru-uc.ac.jp
}

\begin{abstract}
Progress of ubiquitous computing oriented ICT promotes development of information appliances with various functions. In spite of the progress, most of user cannot receive the benefit of them. In this paper as the reason of it, the problems related to increasing enormous amount of information contents were discussed. Based on the problems, there are new challenges for designing user experiences; interactions with vast amount of information contents and integrations ubiquitous computing applications into daily life. Analyzing these challenges from view of human-centered design could derive the necessity to services design based on ubiquitous technologies and validation of them.
\end{abstract}

Keywords: Ubiquitous computing, user experience, information content, information appliance

\section{Introduction}

Recently "ubiquitous computing" is used in the context that micro-computers are embedded in various artifacts and user can receive various kinds of the benefits by their connecting each other. Japanese Government has published white paper which included life styles under ubiquitous computing [1]. It was called u-Japan policy whose life style seems to be the world through rose-colored glasses. Furthermore, for digital device manufactures ubiquitous-computing oriented information appliances are expected as new market break-through. However the progress of ICT like ubiquitous computing are not always welcomed for users. According to NRI report [2], most of Japanese people feel anxiety that they could not master the new appliances. Before receiving benefits from ubiquitous computing properly which are expected by computer scientists or engineers, some problems should be overcome.

For examples, we are facing the following problems. Firstly problem is that some people must arm with digital appliances. Progress of ICT promotes to succeed to implement various functionalities to small devices. Consequently we are surrounded by many appliances such as mobile phone, digital camera, USB memory, MP3 audio 
device, PDA and so on. Some businessmen must always have some devices among them.

In contrast to above situation, some functions of computing tend to be concentrated on mobile phones. A lot of function does not always correlates to usability commonly. Actually some functions are not used at all for some people. Recently secure specification is strongly required. The stronger the security is the less easy the usage is. Consequently concentration of function does not yield conveniences of appliances.

Regarding information contents, amount of contents a person can handle in daily life is increasing rapidly based on advance of storage technology. At the same time, way of contents usage is getting various. As a result, users would be puzzled the usage and then the contents would be scattered like rubbish. Actually most of graphical contents by digital camera are said to remain unused.

Aside from the original concept of ubiquitous computing [3], its general trends would begin with device based concept embedding computer and anytime, anywhere network concept. Compared with developments of a large number of new digital devices, quite a few can be acceptable for user. As mentioned above, most of people in Japan are not necessary to have new devices in daily life. To bridge gaps between technological trend and user's necessity, these problems should be challenged in view of human-centered design. In this paper, a problem involving relations between user and information content is considered. As illustrating precedent cases, some implications of requirements for designing ubiquitous computing applications in the daily life are discussed from human-centered viewpoints.

\section{Challenges to Vast Amount of Information Contents}

Digital devices such as digital camera or hard disk recorder can promote to collect information contents. For examples, when we take photos with film camera, the number of photos per a film is limited from twelve to thirty six. On the contrary, we can take hundreds of photo with digital camera. Thus, amount of information contents and usage which user must handles is increasing rapidly as mentioned above. Thus, user has to manage vast amount of information contents as well as use the devices.

Furthermore the contents tend to be scattered in various devices. Regarding digital video, hard disk recorder can store it, personal computer can store it and mobile player device such as iPod can store it. The digital video has scattered in various devices at home. If he/she wants to watch a video, he/she must go to a device where the video might be stored and seek the video.

To solve the situation where information contents are scattered in various devices, there are some projects to set up platform for interoperable use of all digital contents at home. DLNA (Digital Living Network Alliance) [4] is a representative project for IT related leading industries (Fig.1). First DLNA project points out that we live in three digital islands at home; (1) the PC Internet world where PC and PC peripherals communicate, (2) the broadcast world of set-top boxes and traditional 
consumer electronics, (3) the mobile world of multimedia phones, PDAs, laptop computers and similar devices provides unparalleled connectivity and freedom of movement into and out of the home environment. User wants to access the information contents without regard to devices in these three domains. But the expectations have largely been unfulfilled under the present circumstances. To fulfill the expectations an interoperability framework is proposed, which will manage and distribute rich digital content to devices such as TVs and wireless monitors from devices such as digital stills cameras, camcorders and multimedia mobile phones. The framework is expected to define interoperable building blocks for devices and software infrastructure. It should cover physical media, network transports, media formats, streaming protocols and digital rights management mechanisms.

DLNA concept is itself excellent idea, however that accelerates the increase of information contents user can enjoy and must manage.

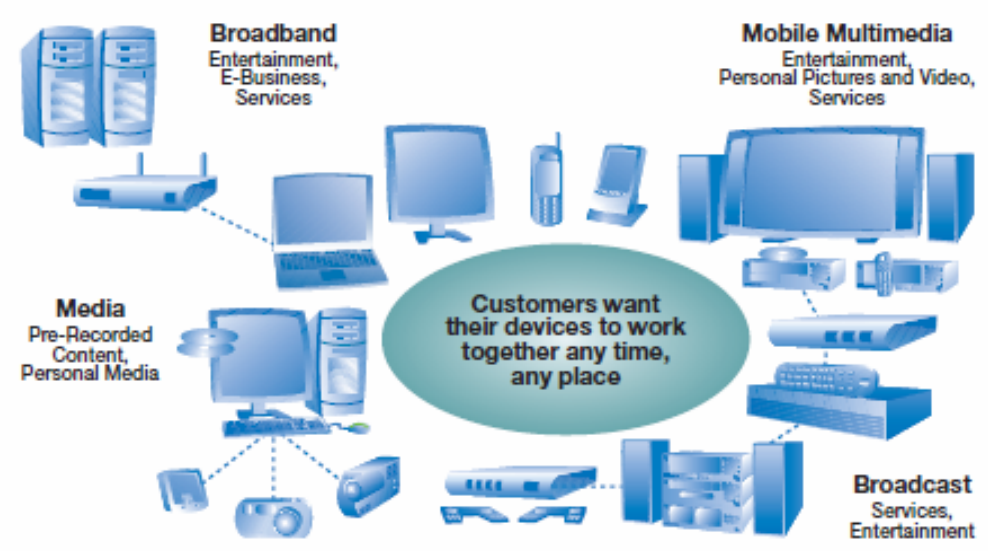

Fig. 1. DLNA Vision (Use Case Scenarios, White Paper, Digital Living Network Alliance 2004)

\section{Contents Search in Daily Life}

As mention above, it is definitely inevitable to interact with vast amount of information contents in daily life. Therefore some challenges in relation to the contents should be sorted out.

First, the effective information search should be invented to browse vast amount of contents. Technically in order to raise performance of information search the information should be restructured properly. If user interface based on the restructuring is implemented into devices, the system requires user to understand the 
structure. As a result it would be uneasy and often dull to search information in daily life. User interface of the contents search should be considered from user view so that search activity itself may be fun. XMB by Sony Inc is one of options among pleasant user interfaces.

Regarding original contents such as digital photos or digital movies, their search methods would be restricted. Because effective content attribute in searching is just only date or time. The contents category is limited to only time without adding new attribution by users themselves. Thus design of user interface for exploring contents would be restricted.

Second challenge is issue of development of new application using contents. In case that the information contents consist of analog data, it is very difficult to separate the contents from the media which store them. For instance, our experiences of hearing music with LP record are limited in living room or any room where the stereo player exists. If we need to copy music in LP record into cassette tape, we had to connect stereo player with tape recorder. After the contents were digitalized, it has become easy to separate them from media and easy to be moved between media. Now we can focus the contents themselves and invent various way of enjoyment. Not only in PC but also in hard disk recorder as home appliance, various types of digital contents such as digital movie, digital image and digital music file and so on are stored in a same device or in home network together.

Each digital information contents need to be easy to be handled and to be managed by each software application. If the content is compiled easily, user can enjoy new contents experience. For simple examples, we could experience ourselves "Best 25 music" or "80's music" through iPod's use. As another example, "x-Pict story" application in Sony hard disk recorders can support to make an original movie by compiling digital photos and music stored in the appliance.

In order to promote user to accept and use these applications they need to be understood easily about their main way of use and their impacts in user lifestyle context. In this sense it is quite important to represent enjoyable service images of the contents use and to develop easy to use user interfaces.

\section{Incorporation into daily life}

Many researches and developments related to technical issues based on ubiquitous computing and networking have been conducted. For example UbiLab [5] project is aiming to develop fundamental and application software for the ubiquitous computing environment. Ubila [6] project which consists of academic-industrial cooperation covers total system for supporting ubiquitous network and services, including core network, access network and ubiquitous end objects.

ICE-CREAM [7] project focused on the contents programs in end-user home environment. The objectives are to investigate the potential of new technologies for designing new concepts for interactive and enhanced broadcast, to create programs and environments in which users can interact with the content of the programs and 
compose personal programs to create personal flavor and emotion. The project investigated how to make compelling experiences for end users based on the possibilities of integrating technologies for interactive media, for example, DVBMHP, MPEG-4, 3D graphics and Internet technologies. Technological options that address different levels of interactivity for end-users were investigated and implemented in prototypes, and supported by business frameworks.

Approach of these projects is typical technology centered although ICE-CREAM project conducted user testing. From technical viewpoint, ubiquitous computing can promise to offer immersive and exciting experience, however our daily life does not need always so stimulated. The point is whether user experiences base on those technologies can accustom users' way of life or not. The relevancy of new user experiences to our daily life should be considered to implement ubiquitous computing application.

In order to understand the relevancy between new technology experience and lifestyle habit, our research project conducted an experiment of how people merge Internet use in their habit of TV watching. The experiment was performed in situations which are close to informants' actual TV watching lifestyle in their living room.

As a result there were two patterns of browsing web site in watching TV; one pattern was that informants frequently browsed before and after watching TV. Before they decided the TV program they intend to watch, they repeated browsing and zapping by turns. Another pattern was that informant occasionally browsed and zapped at intervals to the end of test (Fig. 2).

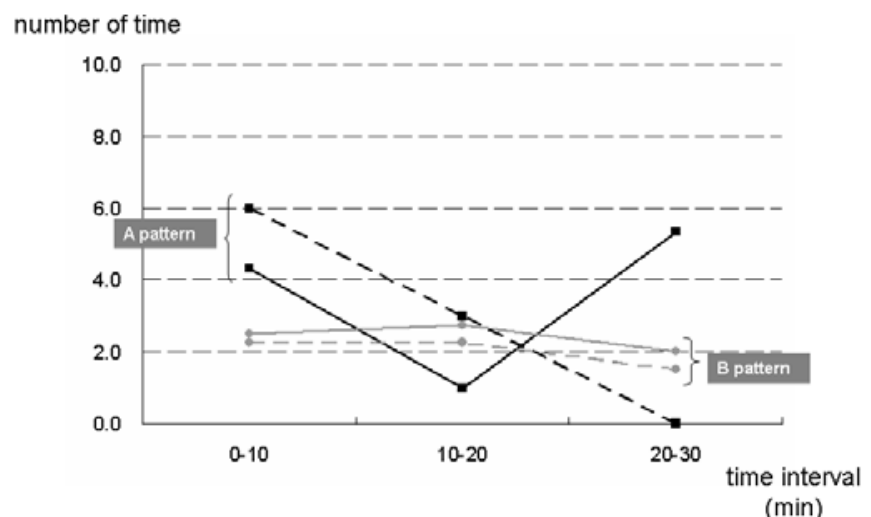

Fig. 2. Number of web access (full line) and zapping of TV channel (dot-line)

Watching TV programs are not always similar to browsing web site in view of cognitive interaction between user and contents, that is, the style of TV watching is mostly passive, whereas web site's one is active. This means it would be inconvenient to unite those two functions into a system such as TV with Web browsing or PC with TV tuner. But both of those activities work closely together although there are several 
patterns of them. This shows possibilities of new type of media environments including more than two devices that have those functions separately.

Through these results, the following implications could be shown at least.

First, the design scope of ubiquitous computing system should be extended to way of life context. It is difficult to specify requirements for the system only from user interaction with the system. The design must be begun with service definition which identifies relation between user and the information contents properly in the context of way of life.

Moreover, renewed additional criterions to present usability's criteria need to validate the service design. Actual installment of the ubiquitous computing system in the lifestyle context need not only fun or excitement viewpoint but also natural or piece and quite viewpoint. In particular habituation of using a system would be so important that the system might need to establish the way of life.

In order to integrate ubiquitous application into way of life and clarify the criterions for validation of new system, log data from actual human life style must be required. There have been some projects to monitor and log user activities. "KoKomemo" is one of logging systems with mobile phones with camera for user problems in town. It was developed in "Yaorozu" project [8] in Japan. The system can capture problems scenes in daily life.

But it is not sufficient to know total user context of lifestyle. PlaceLab [9] project conducts research by designing and building real living environments - "living labs" - that are used to study technology and design strategies in context. It is a residential condominium, designed to be a highly flexible and multi-disciplinary observational research facility for the scientific study of people and their interaction patterns with new technologies and home environments.

\section{Conclusions}

Recently amount and variety of digital information content is increasing rapidly. Their amount and diversity become to affect users experiences and their life style at home. Better acceptances of ubiquitous applications need to solve those problems. The solution could be inferred from not only user experiences analysis but also real services definition in context of daily life style. Furthermore the services should be validated for user to receive actual benefits.

Essentially for designing ubiquitous application we had better begin with building concepts of those services. Although we could recognize the importance, we must face undeveloped methodologies for design and validation of the services mentioned above. In parallel with technological projects, human-centered or multidisciplinary projects need to be conducted to yield those methodologies especially in Japan. 


\section{References}

1. The Ministry of Internal Affairs and Communications in Japan, u-Japan Policy (2004)

2. Nomura Research Institute, “Cyber Life Observation” (2002)

3. Weiser, M., Brown, S.J., The Coming Age of Calm Technology, Beyond Calculation -- The Next Fifty Years of Computing Edited by Peter J. Denning, Robert M. Metcalfe, p75-85, Springer-Verlag (1997)

4. Overview and Vision White Paper, Digital Living Network Alliance (2004)

5. UbiLab, http://www.ht.sfc.keio.ac.jp/ubi-lab/ (2006)

6. Ubilia, http://www.ubila.org/e/e_index.html (2006)

7. ICE-CREAM-phr-0404-01/Janse, The ICE-CREAM Project Final Report, Deliverable D22 (2004)

8. Yaorozu, http://www.8mg.jp/en/index.html (2005)

9. PlaceLab, http://architecture.mit.edu/house_n/placelab.html (2004) 